\title{
Regarding the distribution of glue in the pion
}

\author{
Lei Chang ${ }^{1, *}$ and Craig D. Roberts ${ }^{2,3, \dagger}$ \\ ${ }^{1}$ School of Physics, Nankai University, Tianjin 300071, China \\ ${ }^{2}$ School of Physics, Nanjing University, Nanjing, Jiangsu 210093, China \\ ${ }^{3}$ Institute for Nonperturbative Physics, Nanjing University, Nanjing, Jiangsu 210093, China
}

(Dated: 2020 June 15)

\begin{abstract}
Understanding why the scale of emergent hadron mass is obvious in the proton but hidden in the pion may rest on mapping the distribution functions (DFs) of all partons within the pion and comparing them with those in the proton; and since glue provides binding in quantum chromodynamics, the glue DF could play a special role. Producing reliable predictions for the proton's DFs is difficult because the proton is a three valence-body bound-state problem. As sketched herein, the situation for the pion, a two valence-body problem, is much better, with continuum and lattice predictions for the valence-quark and glue DFs in agreement. This beginning of theory alignment is timely because experimental facilities now either in operation or planning promise to realise the longstanding goal of providing pion targets, thereby enabling precision experimental tests of rigorous theory predictions concerning Nature's most fundamental Nambu-Goldstone bosons.
\end{abstract}

1. Introduction. - The proton was discovered in 1917 [1]. It is stable: looking back through the approximately 14billion year history of the Universe, no proton in isolation has ever been seen to decay. This is one of the most profound features of Nature; and for science it means, inter alia, that protons can readily be used as targets or accelerated as probes for the exploration of other material [2]. These properties have been very profitably exploited, enabling the discovery of quarks [3-5] and fostering the development of quantum chromodynamics (QCD) as the theory of strong interactions [6].

When discussing proton structure, parton distribution functions (DFs) are often the focus [7]. Each DF, $p(x ; \zeta)$, describes the number density of a given parton type, $p=$ valence-quark $(q)$, sea-quark $(\mathcal{S})$, or glue $(g)$, within the proton as a function of the light-front fraction, $x \in(0,1)$, of the proton's momentum that it carries at a resolving scale, $\zeta$. As with many quantities in quantum field theory, like the coupling and masses of elementary fields, these densities depend on the energy scale with which they are probed. Crucially, under certain kinematic conditions [7], such as those prevailing in deep inelastic scattering (DIS) or Drell-Yan (DY) reactions [8, 9], DFs can be extracted from the data acquired.

Since the first empirical DIS studies, a prodigious number of experiments has been completed and analysed with this purpose [10-13]. The current status may be described as follows. (i) The wealth of data has enabled development of numerous QCD-related proton DF fits, which are in fair agreement amongst themselves on the domains favoured by dense data. On the complementary domains, the fits can disagree markedly, with significant physics impact, such as uncertainty in the value of the large- $x$ exponent on the proton's valence-quark distributions [14-17]. (ii) With phenomenology thus positioned, there is great need for rigorous QCD-connected

\footnotetext{
* leichang@nankai.edu.cn

$\dagger$ cdroberts@nju.edu.cn
}

theory input; but even after roughly fifty years of $\mathrm{QCD}$, this is lacking. Many models have been used to compute valence-quark DFs, but similarities and disagreements speak primarily about the practitioners' assumptions; few predictions are available from realistic solutions of the continuum three-valence-body bound-state problem in QCD [18, 19]; results from lattice-regularised QCD (1QCD) are not yet of sufficient precision to materially contribute to the improvement of DF fitting [20]; and there are no calculations of the proton's glue distributions. Plainly, even in the optimal case supplied by the proton, one cannot claim understanding of its DFs.

Whereas the proton is defined by its valence $u+u+d$ quark content, mesons are a different form of hadron matter, being constituted from a valence-quark and -antiquark. The pion has been a known hadron for more than seventy years [21, 22]. Like all mesons, pions are unstable; and while the charged states, $\pi^{+}=u \bar{d}$ ( $u$-quark $+d$-antiquark) and $\pi^{-}=d \bar{u}$, decay only via weak interactions, they do not live long enough to serve readily as targets. Hence, regarding parton DFs within the pion, the empirical situation is dire.

Information on the pion's valence-quark DF was obtained in a series of pion-beam experiments at CERN [23-25] and Fermilab (E615) [26] more than thirty years ago and also using the Sullivan process [27] (scattering from the proton's pion cloud) at DESY twenty years ago $[28,29]$. Phenomenological fits of these data [30-34] yield DFs with notable mutual dissimilarities [35] and, excepting Ref. [30], possessing large- $x$ behaviour in conflict with predictions derived from QCD. Specifically [36, Sec. 5A]: at any resolving scale, $\zeta$, for which data may be interpreted in terms of DFs, the pion's valence-quark DF is predicted to exhibit the following behaviour:

$$
q^{\pi}(x ; \zeta) \stackrel{x \cong 1}{=} c(\zeta)(1-x)^{\beta_{\pi}(\zeta)}, \beta_{\pi}(\zeta)>2,
$$

$c(\zeta)$ is a constant, i.e. independent of $x$. Contradicting this, the $x \simeq 1$ behaviour of the phenomenological DFs in Refs. [31-34] corresponds to $\beta_{\pi}(\zeta) \approx 1$.

A key difference between the study of proton and pion 
DFs is that QCD theory has made real progress with the pion during the past decade. Preceding this period, numerous models had been used to compute the pion's valence-quark DF [10], with the outcome depending on the model chosen. Today, a unified understanding has been provided for those diverse results [36]; new models are being explored [37-41]; and continuum and lattice studies are delivering parameter-free predictions, with results even for the pion's glue distribution [42-44]. Complementing these theory developments, new-generation facilities are developing the capacity to test the predictions via the Sullivan process [27] in high-luminosity electron+proton collisions $[19,45-48]$ or directly with highenergy, high-intensity pion and kaon beams [49].

2. Hadron Scale Pion Distributions. - In the Standard Model, the pion is a bound state, just like the proton. The only mechanical difference is that the pion emerges as a pole in the quark+antiquark scattering matrix whereas the proton appears in the study of three-quark scattering. This perspective defines both modern continuum and lattice treatments, e.g. Refs. [50, 51]. It replaces earlier model notions that linked the pion with a longwavelength fluctuation of a chiral condensate [52].

Pion properties can be studied using continuum Schwinger function methods (CSMs) [53-56]. Here, QCD's gap and Bethe-Salpeter equations are central. They have been used to elucidate connections between the pion's Bethe-Salpeter amplitude and light-front wave function [57], leading to the prediction that at the hadron scale, $\zeta_{H}$, with accuracy that will not be exceeded in foreseeable experiments, the pion's leading-twist two dressedparticle distribution amplitude (DA), $\varphi_{\pi}$, and valencequark DF are related as follows [36, Sec. $3 \mathrm{~A}]$ :

$$
q^{\pi}\left(x ; \zeta_{H}\right) \approx \varphi_{\pi}^{2}\left(x ; \zeta_{H}\right) / n_{\varphi},
$$

$n_{\varphi}=\int_{0}^{1} d x \varphi_{\pi}^{2}\left(x ; \zeta_{H}\right)$. Consequently, $\zeta_{H}$ is the scale at which the dressed-quark and -antiquark, produced by QCD's gap equation, carry all properties of the pion, including baryon number and momentum:

$$
\int_{0}^{1} d x q^{\pi}\left(x ; \zeta_{H}\right)=1=\int_{0}^{1} d x 2 x q^{\pi}\left(x ; \zeta_{H}\right) .
$$

The pion's sea and glue distributions are zero at $\zeta_{H}$. (In the $\mathcal{G}$-parity symmetry-limit [58], a good approximation in Nature, the $\pi^{+}$is characterised by a single valence distribution: $q^{\pi}\left(x ; \zeta_{H}\right)=u^{\pi}\left(x ; \zeta_{H}\right)=\bar{d}^{\pi}\left(x ; \zeta_{H}\right)$.)

Eq. (2) is important because, following thirty years of controversy, the past decade has seen a clear picture of the pion DA emerge $[42,43,57,59-65]: \varphi_{\pi}$ is a concave function, which is simultaneously dilated and reduced in maximum magnitude relative to its asymptotic profile $\varphi_{\text {as }}(x)=6 x(1-x)$ [66-68]. Both features owe to the phenomenon of emergent hadron mass [69], the likely explanation for $>98 \%$ of the visible mass in the Universe.

Against this canvas, informed by gap and BetheSalpeter equation solutions obtained with refined kernels
[70, 71], Ref. [43] presented a parameter-free prediction for the pion's valence-quark DF:

$$
\begin{aligned}
& q^{\pi}\left(x ; \zeta_{H}\right)=375.32 x^{2}(1-x)^{2} \\
& \quad \times[1-2.5088 \sqrt{x(1-x)}+2.0250 x(1-x)]^{2} .
\end{aligned}
$$

Importantly, and distinct from modelling and data fitting, the hadron (initial) scale is not introduced as a parameter in Ref. [43]. Instead, the value of $\zeta_{H}$ is a prediction derived from the behaviour of QCD's processindependent (PI) effective charge [72, 73].

QCD's PI charge is accurately interpolated by the following function [43]:

$$
\hat{\alpha}\left(k^{2}\right)=\frac{\gamma_{m} \pi}{\ln \left[\frac{\mathcal{K}^{2}\left(k^{2}\right)}{\Lambda_{\mathrm{QCD}}^{2}}\right]}, \mathcal{K}^{2}(y)=\frac{a_{0}^{2}+a_{1} y+y^{2}}{b_{0}+y},
$$

$\gamma_{m}=4 /\left[11-(2 / 3) n_{f}\right], n_{f}=4, \Lambda_{\mathrm{QCD}}=0.234 \mathrm{GeV}$, with (in $\mathrm{GeV}^{2}$ ): $a_{0}=0.104(1), a_{1}=0.0975, b_{0}=0.121(1)$. Here, to simplify comparisons with other sources, the interpolation is expressed through $\Lambda_{\mathrm{QCD}}$, the mass-scale introduced in perturbation theory via the process of regularisation and renormalisation required in defining fourdimensional quantum field theories.

$\hat{\alpha}\left(k^{2}\right)$ agrees to better than $0.1 \%$ with perturbative QCD's one-loop coupling on $k^{2} \gtrsim\left(9 \Lambda_{\mathrm{QCD}}\right)^{2}$. That perturbative coupling exhibits an unphysical (Landau) pole at $k^{2}=\Lambda_{\mathrm{QCD}}^{2}$ [74]; but in deriving $\hat{\alpha}\left(k^{2}\right)$, the Landau pole is seen to be eliminated by nonperturbative gauge-sector dynamics $[72,73]$. Comparing with the perturbative expression, $k^{2} / \Lambda_{\mathrm{QCD}}^{2} \rightarrow \mathcal{K}^{2}\left(k^{2}\right) / \Lambda_{\mathrm{QCD}}^{2}$ as the logarithm's argument in Eq. (5). The value $m_{G}:=$ $\mathcal{K}\left(k^{2}=\Lambda_{\mathrm{QCD}}^{2}\right)=0.331(2) \mathrm{GeV}$ defines a screening mass. It marks a boundary: the coupling alters character at $k \simeq m_{G}$ so that modes with $k^{2} \lesssim m_{G}^{2}$ are screened from interactions and the theory enters a practically conformal domain [75-78]. The line $k=m_{G}$ separates longand short-wavelength physics; hence, serves as the natural definition for the hadron scale, viz. $\zeta_{H}=m_{G}$.

3. Pion Distributions at $\zeta=2 \mathrm{GeV}$. - DFs and/or their moments are commonly quoted at $\zeta=\zeta_{2}=2 \mathrm{GeV}$; and one may obtain the DFs at $\zeta_{2}$ via evolution from another scale by integrating the DGLAP equations [79-82]. When completing this exercise, a prescription must be specified because the standard DGLAP equations involve QCD's running coupling. In modelling and fitting, it is usual to adopt a purely perturbative-QCD perspective, implementing evolution with a DGLAP kernel calculated at a given order in perturbation theory. If the scale at which evolution begins is large enough, then leadingorder (LO) evolution kernels may be adequate, at least in practice. If they fail, then next-to-leading-order (NLO) can be implemented, and so on, in principle.

A different approach is advocated in Refs. [43, 73]. Namely, adapting ideas from Refs. [74, 85, 86], evolution is implemented by using $\hat{\alpha}\left(k^{2}\right)$ in Eq. (5) to integrate 


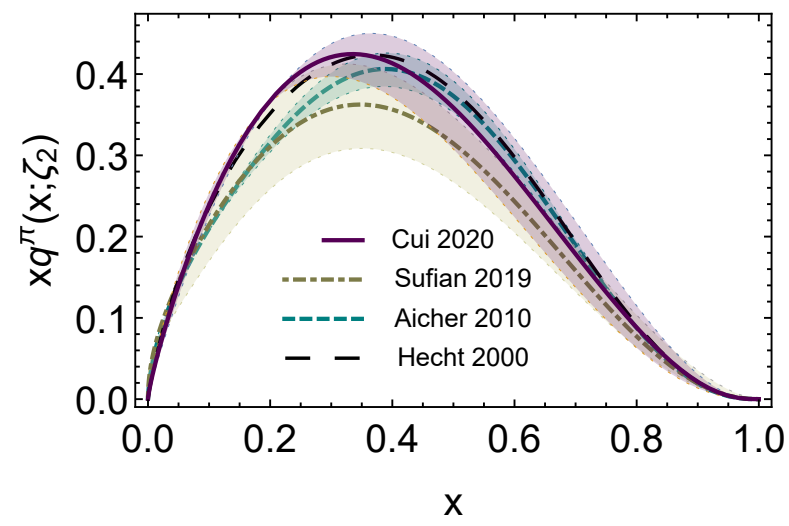

FIG. 1. $x q^{\pi}\left(x, \zeta_{2}=2 \mathrm{GeV}\right)$, pion valence-quark distribution: solid purple curve, prediction from Ref. [43]. The associated band expresses a conservative estimate of uncertainty in the prediction, obtained by varying $\zeta_{H}$ by $\pm 10 \%$. Comparisons: dot-dashed olive-green curve, lQCD result [83]; short-dashed teal curve and associated uncertainty band, fit to E615 data [30], which incorporated next-to-leading-logarithm effects in the hard-scattering kernel; and long-dashed black curve, original CSM result [84].

the one-loop DGLAP equations. This $\hat{\alpha}$ scheme eliminates ambiguity from the resulting predictions because it renders moot any questions regarding the order of the evolution kernels. Working with the hadron scale DFs described in Sec. 2, one obtains the parameter-free predictions for the $\zeta_{2}=2 \mathrm{GeV}$ valence, sea, and glue DFs drawn in Figs. 1-5. Using those predictions, one calculates the following light-front momentum fractions:

$$
\begin{gathered}
\qquad \begin{array}{r|c|c}
\left\langle 2 x q_{\zeta_{2}}^{\pi}\right\rangle & \left\langle x \mathcal{S}_{\zeta_{2}}^{\pi}\right\rangle & \left\langle x g_{\zeta_{2}}^{\pi}\right\rangle \\
\hline 0.48(4) & 0.11(2) \mid 0.41(2)
\end{array} \\
\text { where }\left\langle x^{n} p_{\zeta_{2}}^{\pi}\right\rangle=\int_{0}^{1} d x x^{n} p^{\pi}\left(x ; \zeta_{2}\right) .
\end{gathered}
$$

Evidently, gluon partons carry a large fraction of the pion's momentum at $\zeta_{2}$.

Given that the calculation of Mellin moments of the pion's valence-quark DF is still the subject of many lQCD studies [87, 88], it is worth recording the following predictions obtained using the pion DFs in Ref. [43]:

$$
\begin{array}{c|c|c}
\left\langle x q_{\zeta_{2}}^{\pi}\right\rangle & \left\langle x^{2} q_{\zeta_{2}}^{\pi}\right\rangle & \left\langle x^{3} q_{\zeta_{2}}^{\pi}\right\rangle \\
\hline 0.24(2) & 0.094(13) & 0.047(08) \\
\left\langle x^{4} q_{\zeta_{2}}^{\pi}\right\rangle & \left\langle x^{5} q_{\zeta_{2}}^{\pi}\right\rangle & \left\langle x^{6} q_{\zeta_{2}}^{\pi}\right\rangle \\
\hline 0.027(05) & 0.017(04) & 0.011(03)
\end{array}
$$

Larger values for the $n \geq 2$ moments indicate a harder $\mathrm{DF}$, i.e. a function with greater support on the valencequark domain than the prediction in Ref. [43]; consequently, less support on the complementary domain.

It is worth continuing with a discussion of the pointwise behaviour of the pion's valence-quark distribution, Fig. 1. Within uncertainties, the parameter-free prediction from

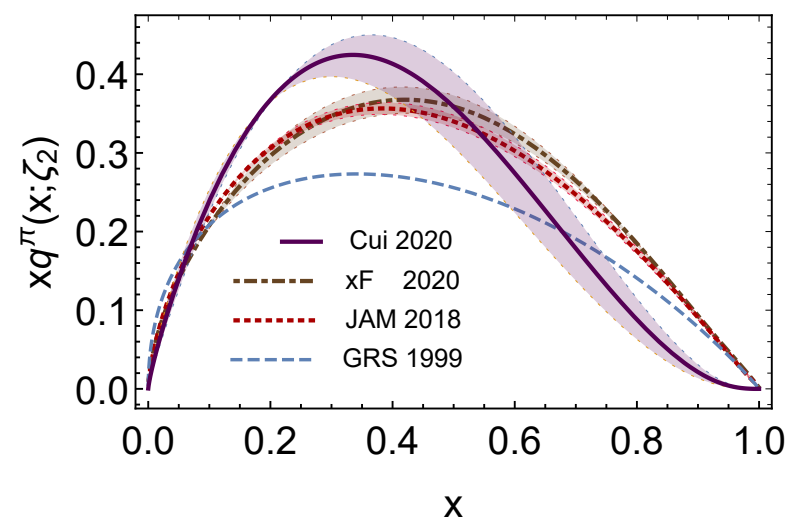

FIG. 2. $x q^{\pi}\left(x, \zeta_{2}=2 \mathrm{GeV}\right)$, pion valence-quark distribution: solid purple curve and associated uncertainty band, prediction from Ref. [43]. Comparisons are selected fits to data: dashed blue curve, [32]; dotted red curve and associated uncertainty band, [33]; dot-dashed brown curve and band, [34].

Ref. [43] agrees with the IQCD result in Ref. [83]; and both agree well on the valence-quark domain with the NLO analysis of E615 data [26] described in Ref. [30], which is the only work thus far to include next-to-leadinglogarithmic (NLL) threshold resummation effects when calculating the hard-scattering kernel. (N.B. Ref. [30] used sea and glue distributions from Ref. [32]; hence, the valence distribution in Ref. [30] is likely less reliable on $x \lesssim 0.4$.) Interestingly, the result in Ref. [84], which reignited debate over the pion's valence-quark DF and was obtained using algebraic Ansätze for the elements in the pion wave function, also agrees with the modern CSM prediction. The continuum prediction [43], 1QCD result [83], NLO+NLL fit [30], and QCD-based model [84] are consistent with Eq. (1). (See also Ref. [43, Fig. 5a].)

On the other hand, NLL effects were omitted in producing the comparison fits [32-34] drawn explicitly in Fig. 2, which display some notable mutual differences and are also in conflict with Eq. (1). Since the fits in Refs. [3234] are harder (possess greater support) at large- $x$ than the prediction from Ref. [43], then one can anticipate that they must also produce harder sea and glue distributions because the valence distribution serves as a source for both sea and glue. Equally, owing to momentum conservation, the data fits must possess less support at low $x$. These expectations were confirmed in Ref. [43, Fig. 4b] and the results are highlighted by Figs. 3, 4 herein.

Regarding Fig. 4, it is worth emphasising that at $\zeta=\zeta_{2}$ the pion's predicted glue distribution is larger in magnitude than its valence distribution on $x \lesssim 0.22$. Such an outcome was to be expected. Indeed, as stated in connection with Eq. (5), at $\zeta=\zeta_{H}$ all properties of the pion are expressed in the dressed-quark and -antiquark degreesof-freedom that are generated dynamically in solving the continuum bound-state problem. Under evolution to $\zeta>\zeta_{H}$, these quasiparticles steadily shed their cladding, thereby producing populations of sea and glue partons. 


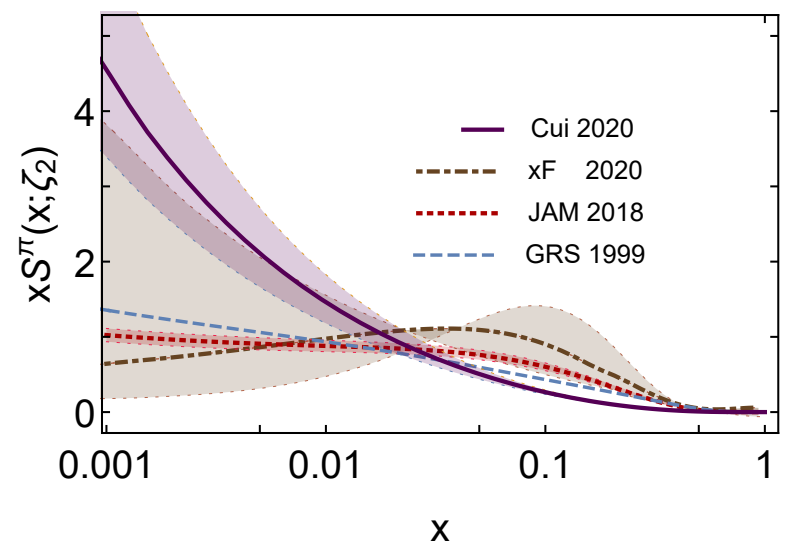

FIG. 3. Sea distribution, $x \mathcal{S}^{\pi}\left(x, \zeta_{2}=2 \mathrm{GeV}\right)$ : solid purple curve, prediction from Ref. [43]. The associted band expresses a conservative estimate of uncertainty in the prediction, obtained by varying $\zeta_{H}$ by $\pm 10 \%$. Comparisons are selected fits to data: dashed blue curve, [32]; dotted red curve and associated band, [33]; dot-dashed brown curve and band, [34].

The size of these populations increases with increasing $\zeta$. Hence, all symmetry-preserving treatments of pion structure and dynamics must produce nonzero valence, sea, and glue DFs at any scale for which an analysis of experiments may be interpreted in terms of DFs. Furthermore, there is no scale at which all three vanish identically.

Figs. $1-4$ show that the pointwise form of each DF obtained via a fit to data which omits NLL effects disagrees markedly with the comparable CSM prediction [43]. This stresses the need for both improved data analyses ${ }^{1}$ and additional rigorous DF predictions from QCD theory.

In this connection, recalling Fig. 1, the CSM prediction for the pion's valence-quark DF [43] is confirmed by the lQCD result in Ref. [83].

No lQCD results are available for the pion's sea distribution; so, nothing can yet be directly concluded on this score. Regarding phenomenology, Fig. 3 highlights that the pion's sea distribution is very poorly constrained by existing data analyses.

On the other hand, lQCD results for the pion's glue DF have recently become available [44]. They are compared in Fig. 5 with the CSM prediction [43]: within uncertainties, there is pointwise agreement between the two results on the entire depicted domains. Combined with similar agreement between CSM and IQCD predictions for the pion's valence distribution (Fig. 1), one has evidence to suggest that the CSM prediction for the pion's sea distribution, Fig. 3, is also reliable.

\footnotetext{
${ }^{1}$ Complementing Ref. [30], the potential impacts of including NLL effects when extracting DFs from data are also illustrated in Ref. [89], e.g. all DFs are softer at large- $x$ and a greater fraction of the pion's light-front momentum is carried by glue, with the cost paid largely by the sea fraction.
}

A
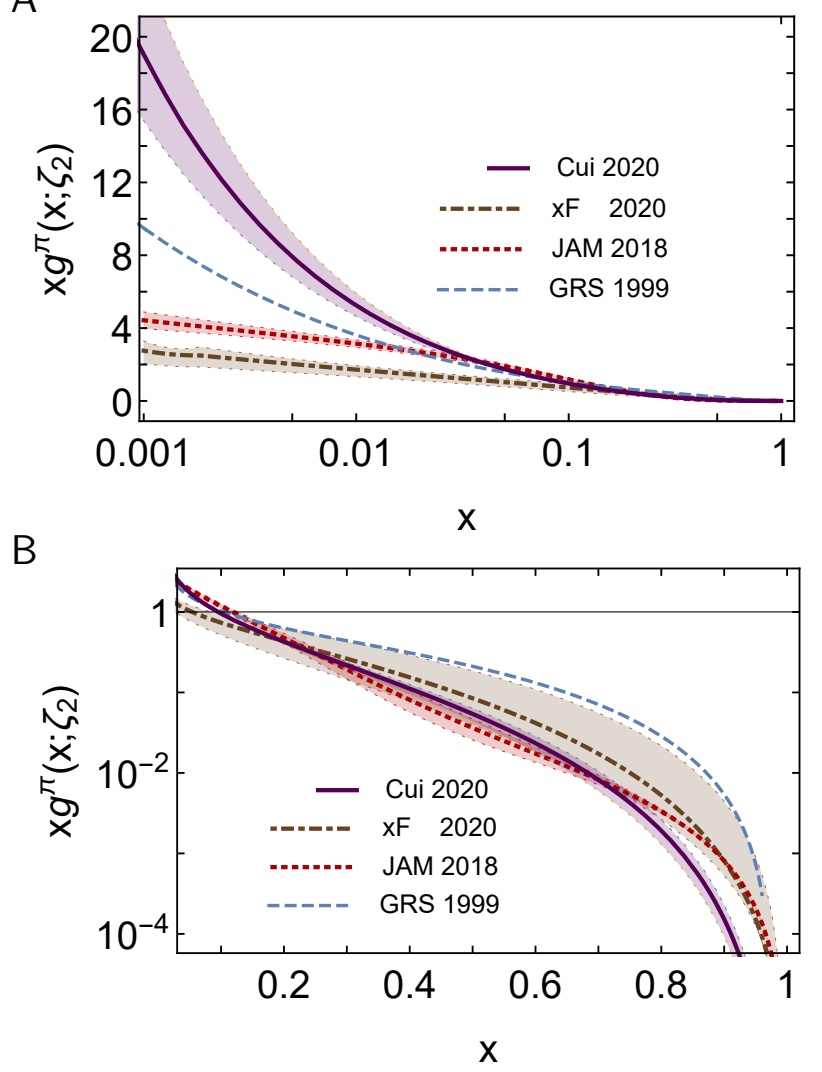

FIG. 4. Glue distribution, $x g^{\pi}\left(x, \zeta_{2}=2 \mathrm{GeV}\right)$ : solid purple curve, prediction from Ref. [43]. Panel A highlights low- $x$ and Panel B, large- $x$. The band surrounding this curve expresses a conservative estimate of uncertainty in the prediction, obtained by varying $\zeta_{H}$ by $\pm 10 \%$. Comparisons are selected fits to data: dashed blue curve, [32]; dotted red curve and associated band, [33]; dot-dashed brown curve and band, [34].

Such quantitative agreement between continuum and lattice results for pion DFs supports the perspective on the origin of hadron masses developed in Ref. [90]. Namely, the mass of the pion is much smaller than that of the $\rho$-meson (and the proton) owing to a symmetryensured cancellation in this near Nambu-Goldstone boson between the positive one-body-dressing content of the QCD trace anomaly and the negative binding energy produced by interactions. (See also Ref. [36, Sec. 2 D].)

4. Summary and Perspective. - Through comparisons with continuum and lattice predictions for pion distribution functions (DFs), evidence is accumulating which indicates that the pion DFs inferred from analyses of existing data are pointwise inaccurate, possessing too much support on the valence-quark domain $(x \gtrsim 0.2)$ and too little on its complement. Additional evidence suggests that the discrepancies may be ameliorated, or even eliminated, by inclusion of next-to-leading-logarithmic threshold resummation effects in the hard-scattering kernels whose accurate representation is an essential element in 

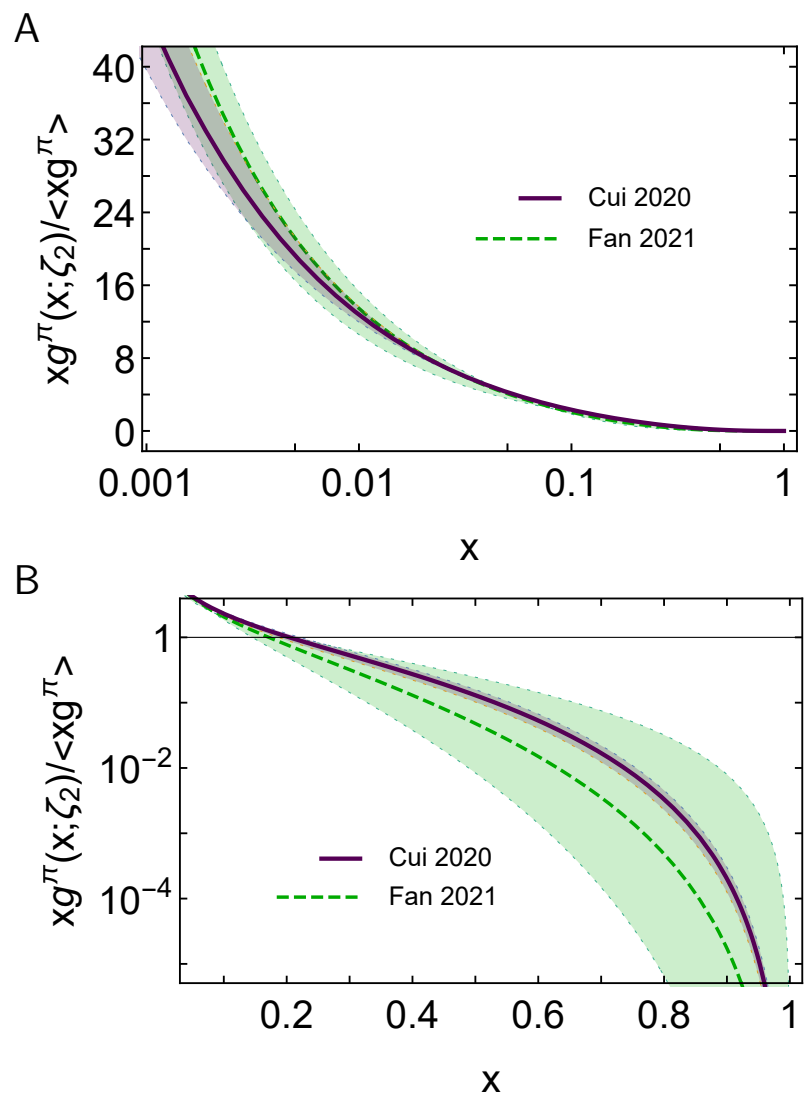

FIG. 5. $\quad x g^{\pi}\left(x, \zeta_{2}\right) /\left\langle x g_{\zeta_{2}}^{\pi}\right\rangle, \zeta_{2}=2 \mathrm{GeV}$, normalised glue distribution: solid purple curve, prediction from Ref. [43]. Panel A highlights low- $x$ and Panel $\mathrm{B}$, large- $x$. Comparison curve: lQCD result [44], for which the associated band expresses aspects of statistical error and systematic uncertainty associated with using an assumed functional form to fit the results.

the determination of DFs through data fitting. If these remarks are correct, then their implications for the DFs of nucleons and nuclei should similarly be considered; especially because imperfect extractions of such DFs may obscure or provide misleading signals of physics beyond the Standard Model. In any event, one must look forward to the era in which sound QCD-connected predictions are exploited in providing material constraints on data analyses. This time is approaching for pions and kaons.

As Nature's most basic Nambu-Goldstone bosons, pions and kaons are unique. Being mesons quark+antiquark bound-states - they also typify a form of hadron matter about whose structure very little is empirically known. That is changing, with new-generation facilities, in operation or planning, having the capacity to provide unprecedented access to these systems as targets.

Critically, theory is also making rapid progress, beginning to deliver robust predictions for the measurable properties of pions and kaons. So, it is reasonable to expect that sometime after the centenary of the prediction of the pion's existence [21] and before the centenary of its discovery [22], science will finally have the information necessary to draw charts which reveal the pion's structure in exquisite detail. This may finally explain why, inter alia, basic features of emergent hadron mass are hidden in the pion whereas they are blatant in the proton.

Acknowledgments. - We are grateful to Z. Fan and H.$\mathrm{W}$. Lin for providing us with information on their simulation results; to J. Lan and Z.-B. Xing for assistance with running DGLAP evolution codes; and for constructive comments from V. Andrieux, D. Binosi, W.-C. Chang, Z.-F. Cui, O. Denisov, M. Ding, R. Ent, F. Gao, T. Horn, W.-D. Nowak, S. Platchkov, C. Quintans, K. Raya, J. Rodríguez-Quintero and S. M. Schmidt. This work benefited from discussions and presentations during the "Workshop on Pion and Kaon Structure at the EIC", hosted by the Center for Frontiers in Nuclear Science, 2020 Jun. 2-5, and the ongoing series of CERN-hosted workshops on "Perceiving the Emergence of Hadron Mass through AMBER@CERN" - 2019 Dec. 11 (I), 2020 Mar. 30-Apr. 02 (II), 2020 Aug. 06-07 (III), 2020 Nov. 30Dec. 4 (IV), 2021 Apr. 27-30 (V). Research supported by: Government of China Thousand Talents Plan for Young Professionals.
[1] E. Rutherford, Collision of alpha particles with light atoms iv. an anomalous effect in nitrogen, Philosophical Magazine xxxvii (1919) 581-587.

[2] J. D. Cockroft, E. T. S. Walton, Experiments with High Velocity Positive Ions. I. Further Developments in the Method of Obtaining High Velocity Positive Ions, Proc. Roy. Soc. Lond. A 136 (1932) 619-630.

[3] R. E. Taylor, Deep inelastic scattering: The Early years, Rev. Mod. Phys. 63 (1991) 573-595.

[4] H. W. Kendall, Deep inelastic scattering: Experiments on the proton and the observation of scaling, Rev. Mod. Phys. 63 (1991) 597-614.

[5] J. I. Friedman, Deep inelastic scattering: Comparisons with the quark model, Rev. Mod. Phys. 63 (1991) 615629 .
[6] W. J. Marciano, H. Pagels, Quantum Chromodynamics, Nature 279 (1979) 479-483.

[7] R. Brock, et al., Handbook of perturbative QCD: Version 1.0, Rev. Mod. Phys. 67 (1995) 157-248.

[8] J. I. Friedman, H. W. Kendall, R. E. Taylor, Deep inelastic scattering: Acknowledgements, Rev. Mod. Phys. 63 (1991) 629.

[9] S. Drell, T.-M. Yan, Massive Lepton Pair Production in Hadron-Hadron Collisions at High-Energies, Phys. Rev. Lett. 25 (1970) 316-320, [Erratum: Phys. Rev. Lett. 25, $902(1970)]$.

[10] R. J. Holt, C. D. Roberts, Distribution Functions of the Nucleon and Pion in the Valence Region, Rev. Mod. Phys. 82 (2010) 2991-3044.

[11] J. Gao, L. Harland-Lang, J. Rojo, The Structure of the 
Proton in the LHC Precision Era, Phys. Rept. 742 (2018) $1-121$.

[12] D. F. Geesaman, P. E. Reimer, The sea of quarks and antiquarks in the nucleon, Rept. Prog. Phys. 82 (2019) 046301.

[13] J. J. Ethier, E. R. Nocera, Parton Distributions in $\mathrm{Nu}-$ cleons and Nuclei, Ann. Rev. Nucl. Part. Sci. 70 (2020) $43-76$.

[14] R. D. Ball, E. R. Nocera, J. Rojo, The asymptotic behaviour of parton distributions at small and large $x$, Eur. Phys. J. C 76 (2016) 383.

[15] E. Segarra, A. Schmidt, T. Kutz, D. Higinbotham, E. Piasetzky, M. Strikman, L. Weinstein, O. Hen, Neutron Valence Structure from Nuclear Deep Inelastic Scattering, Phys. Rev. Lett. 124 (2020) 092002.

[16] A. Courtoy, P. M. Nadolsky, Testing momentum dependence of the nonperturbative hadron structure in a global QCD analysis, Phys. Rev. D 103 (2021) 054029.

[17] D. Abrams, et al., Measurement of the Nucleon $F_{2}^{n} / F_{2}^{p}$ Structure Function Ratio by the Jefferson Lab MARATHON Tritium/Helium-3 Deep Inelastic Scattering Experiment - arXiv:2104.05850 [hep-ex].

[18] C. D. Roberts, R. J. Holt, S. M. Schmidt, Nucleon spin structure at very high $x$, Phys. Lett. B 727 (2013) 249254.

[19] X. Chen, F.-K. Guo, C. D. Roberts, R. Wang, Selected Science Opportunities for the EicC, Few Body Syst. 61 (2020) 43.

[20] H.-W. Lin, et al., Parton distributions and lattice QCD calculations: a community white paper, Prog. Part. Nucl. Phys. 100 (2018) 107-160.

[21] H. Yukawa, On the interaction of elementary particles, Proc. Phys. Math. Soc. Jap. 17 (1935) 48-57.

[22] C. M. G. Lattes, H. Muirhead, G. P. S. Occhialini, C. F. Powell, Processes involving charged mesons, Nature 159 (1947) 694-697.

[23] M. Corden, et al., Production of Muon Pairs in the Continuum Region by $39.5-\mathrm{GeV} / c \pi^{ \pm}, K^{ \pm}, p$ and $\bar{p}$ Beams Incident on a Tungsten Target, Phys. Lett. B 96 (1980) 417-421.

[24] J. Badier, et al., Experimental determination of the $\pi$ meson structure functions by the Drell-Yan mechanism, Z. Phys. C 18 (1983) 281.

[25] B. Betev, et al., Observation of anomalous scaling violation in muon pair production by $194-\mathrm{GeV} / \mathrm{c} \pi$-tungsten interactions, Z. Phys. C 28 (1985) 15.

[26] J. S. Conway, et al., Experimental study of muon pairs produced by $252-\mathrm{GeV}$ pions on tungsten, Phys. Rev. D 39 (1989) 92-122.

[27] J. D. Sullivan, One pion exchange and deep inelastic electron - nucleon scattering, Phys. Rev. D 5 (1972) 17321737 .

[28] M. Derrick, et al., Observation of events with an energetic forward neutron in deep inelastic scattering at HERA, Phys. Lett. B 384 (1996) 388-400.

[29] C. Adloff, et al., Measurement of leading proton and neutron production in deep inelastic scattering at HERA, Eur. Phys. J. C 6 (1999) 587-602.

[30] M. Aicher, A. Schäfer, W. Vogelsang, Soft-Gluon Resummation and the Valence Parton Distribution Function of the Pion, Phys. Rev. Lett. 105 (2010) 252003.

[31] P. J. Sutton, A. D. Martin, R. G. Roberts, W. J. Stirling, Parton distributions for the pion extracted from DrellYan and prompt photon experiments, Phys. Rev. D 45
(1992) 2349-2359

[32] M. Glück, E. Reya, I. Schienbein, Pionic parton distributions revisited, Eur. Phys. J. C 10 (1999) 313-317.

[33] P. C. Barry, N. Sato, W. Melnitchouk, C.-R. Ji, First Monte Carlo Global QCD Analysis of Pion Parton Distributions, Phys. Rev. Lett. 121 (2018) 152001.

[34] I. Novikov, et al., Parton Distribution Functions of the Charged Pion Within The xFitter Framework, Phys. Rev. D 102 (2020) 014040.

[35] W.-C. Chang, J.-C. Peng, S. Platchkov, T. Sawada, Constraining gluon density of pions at large $x$ by pioninduced $J / \psi$ production, Phys. Rev. D 102 (2020) 054024 .

[36] C. D. Roberts, D. G. Richards, T. Horn, L. Chang, Insights into the Emergence of Mass from Studies of Pion and Kaon Structure - arXiv:2102.01765 [hep-ph], Prog. Part. Nucl. Phys. in press.

[37] G. F. de Teramond, T. Liu, R. S. Sufian, H. G. Dosch, S. J. Brodsky, A. Deur, Universality of Generalized Parton Distributions in Light-Front Holographic QCD, Phys. Rev. Lett. 120 (2018) 182001.

[38] J. Lan, C. Mondal, S. Jia, X. Zhao, J. P. Vary, Pion and kaon parton distribution functions from basis light front quantization and QCD evolution, Phys. Rev. D 101 (2020) 034024

[39] Z.-L. Ma, J.-Q. Zhu, Z. Lu, Quasiparton distribution function and quasigeneralized parton distribution of the pion in a spectator model, Phys. Rev. D 101 (2020) 114005.

[40] L. Chang, K. Raya, X. Wang, Pion Parton Distribution Function in Light-Front Holographic QCD, Chin. Phys. C 44 (11) (2020) 114105.

[41] C. Han, G. Xie, R. Wang, X. Chen, An Analysis of Parton Distribution Functions of the Pion and the Kaon with the Maximum Entropy Input, Eur. Phys. J. C 81 (2021) 302.

[42] M. Ding, K. Raya, D. Binosi, L. Chang, C. D. Roberts, S. M. Schmidt, Symmetry, symmetry breaking, and pion parton distributions, Phys. Rev. D 101 (5) (2020) 054014.

[43] Z.-F. Cui, M. Ding, F. Gao, K. Raya, D. Binosi, L. Chang, C. D. Roberts, J. Rodríguez-Quintero, S. M. Schmidt, Kaon and pion parton distributions, Eur. Phys. J. C 80 (2020) 1064.

[44] Z. Fan, H.-W. Lin, Gluon Parton Distribution of the Pion from Lattice QCD - arXiv:2104.06372 [hep-lat].

[45] C. Keppel, B. Wojtsekhowski, P. King, D. Dutta, J. Annand, J. Zhang et al., Measurement of tagged deep inelastic scattering (TDIS) approved Jefferson Lab experiment E12-15-006.

[46] K. Park, R. Montgomery, T. Horn et al., Measurement of kaon structure function through tagged deep inelastic scattering (TDIS) approved Jefferson Lab experiment C12-15-006A.

[47] A. C. Aguilar, et al., Pion and Kaon Structure at the Electron-Ion Collider, Eur. Phys. J. A 55 (2019) 190.

[48] J. Arrington, et al., Revealing the structure of light pseudoscalar mesons at the electron-ion collider, J. Phys. G 48 (2021) 075106.

[49] V. Andrieux, B. Parsamyan, From COMPASS to AMBER: exploring fundamental properties of hadrons, CERN EP Newsletter 2020/12 - 2021/02.

[50] P. Maris, C. D. Roberts, P. C. Tandy, Pion mass and decay constant, Phys. Lett. B 420 (1998) 267-273.

[51] J. Noaki, S. Aoki, H. Fukaya, S. Hashimoto, T. Kaneko, H. Matsufuru, T. Onogi, E. Shintani, N. Yamada, 
Light meson spectrum with $\mathrm{N}(\mathrm{f})=2$ dynamical overlap fermions, PoS LATTICE2007 (2007) 126.

[52] S. J. Brodsky, C. D. Roberts, R. Shrock, P. C. Tandy, Confinement contains condensates, Phys. Rev. C 85 (2012) 065202.

[53] T. Horn, C. D. Roberts, The pion: an enigma within the Standard Model, J. Phys. G. 43 (2016) 073001.

[54] G. Eichmann, H. Sanchis-Alepuz, R. Williams, R. Alkofer, C. S. Fischer, Baryons as relativistic three-quark bound states, Prog. Part. Nucl. Phys. 91 (2016) 1-100.

[55] V. D. Burkert, C. D. Roberts, Roper resonance: Toward a solution to the fifty-year puzzle, Rev. Mod. Phys. 91 (2019) 011003.

[56] S.-X. Qin, C. D. Roberts, Impressions of the Continuum Bound State Problem in QCD, Chin. Phys. Lett. 37 (12) (2020) 121201.

[57] L. Chang, I. C. Cloet, J. J. Cobos-Martinez, C. D. Roberts, S. M. Schmidt, P. C. Tandy, Imaging dynamical chiral symmetry breaking: pion wave function on the light front, Phys. Rev. Lett. 110 (2013) 132001.

[58] T. D. Lee, C.-N. Yang, Charge Conjugation, a New Quantum Number $G$, and Selection Rules Concerning a Nucleon Anti-nucleon System, Nuovo Cim. 10 (1956) 749-753.

[59] N. G. Stefanis, A. V. Pimikov, Chimera distribution amplitudes for the pion and the longitudinally polarized $\rho$ meson, Nucl. Phys. A 945 (2016) 248-268.

[60] K. Raya, L. Chang, A. Bashir, J. J. Cobos-Martinez, L. X. Gutiérrez-Guerrero, C. D. Roberts, P. C. Tandy, Structure of the neutral pion and its electromagnetic transition form factor, Phys. Rev. D 93 (2016) 074017.

[61] F. Gao, L. Chang, Y.-X. Liu, Bayesian extraction of the parton distribution amplitude from the Bethe-Salpeter wave function, Phys. Lett. B 770 (2017) 551-555.

[62] T. Zhong, Z.-H. Zhu, H.-B. Fu, X.-G. Wu, T. Huang, An improved light-cone harmonic oscillator model for the pionic leading-twist distribution amplitude arXiv:2102.03989 [hep-ph].

[63] J.-H. Zhang, J.-W. Chen, X. Ji, L. Jin, H.-W. Lin, Pion Distribution Amplitude from Lattice QCD, Phys. Rev. D 95 (2017) 094514.

[64] J.-H. Zhang, et al., Kaon Distribution Amplitude from Lattice QCD and the Flavor SU(3) Symmetry, Nucl. Phys. B 939 (2019) 429-446.

[65] G. S. Bali, V. M. Braun, S. Bürger, M. Göckeler, M. Gruber, F. Hutzler, P. Korcyl, A. Schäfer, A. Sternbeck, P. Wein, Light-cone distribution amplitudes of pseudoscalar mesons from lattice QCD, JHEP 08 (2019) 065, [Addendum: JHEP 11, 037 (2020)].

[66] G. P. Lepage, S. J. Brodsky, Exclusive Processes in Quantum Chromodynamics: Evolution Equations for Hadronic Wave Functions and the Form-Factors of Mesons, Phys. Lett. B 87 (1979) 359-365.

[67] A. V. Efremov, A. V. Radyushkin, Factorization and Asymptotical Behavior of Pion Form- Factor in QCD, Phys. Lett. B 94 (1980) 245-250.

[68] G. P. Lepage, S. J. Brodsky, Exclusive Processes in Perturbative Quantum Chromodynamics, Phys. Rev. D 22 (1980) 2157-2198.

[69] C. D. Roberts, On Mass and Matter, AAPPS Bulletin 31 (2021) 6.

[70] L. Chang, C. D. Roberts, Tracing masses of ground-state light-quark mesons, Phys. Rev. C 85 (2012) 052201(R).

[71] D. Binosi, L. Chang, J. Papavassiliou, S.-X. Qin, C. D. Roberts, Natural constraints on the gluon-quark vertex, Phys. Rev. D 95 (2017) 031501(R).

[72] D. Binosi, C. Mezrag, J. Papavassiliou, C. D. Roberts, J. Rodríguez-Quintero, Process-independent strong running coupling, Phys. Rev. D 96 (2017) 054026.

[73] Z.-F. Cui, J.-L. Zhang, D. Binosi, F. de Soto, C. Mezrag, J. Papavassiliou, C. D. Roberts, J. Rodríguez-Quintero, J. Segovia, S. Zafeiropoulos, Effective charge from lattice QCD, Chin. Phys. C 44 (2020) 083102.

[74] A. Deur, S. J. Brodsky, G. F. de Teramond, The QCD Running Coupling, Prog. Part. Nucl. Phys. 90 (2016) 174.

[75] S. J. Brodsky, R. Shrock, Maximum Wavelength of Confined Quarks and Gluons and Properties of Quantum Chromodynamics, Phys. Lett. B 666 (2008) 95-99.

[76] A. C. Aguilar, D. Binosi, J. Papavassiliou, The Gluon Mass Generation Mechanism: A Concise Primer, Front. Phys. China 11 (2016) 111203.

[77] F. Gao, S.-X. Qin, C. D. Roberts, J. Rodríguez-Quintero, Locating the Gribov horizon, Phys. Rev. D 97 (2018) 034010.

[78] M. Q. Huber, Nonperturbative properties of Yang-Mills theories, Phys. Rept. 879 (2020) $1-92$.

[79] Y. L. Dokshitzer, Calculation of the structure functions for deep inelastic scattering and e+ e- annihilation by perturbation theory in quantum chromodynamics. (in russian), Sov. Phys. JETP 46 (1977) 641-653.

[80] V. Gribov, L. Lipatov, Deep inelastic e p scattering in perturbation theory, Sov. J. Nucl. Phys. 15 (1972) 438450 .

[81] L. N. Lipatov, The parton model and perturbation theory, Sov. J. Nucl. Phys. 20 (1975) 94-102.

[82] G. Altarelli, G. Parisi, Asymptotic Freedom in Parton Language, Nucl. Phys. B 126 (1977) 298-318.

[83] R. S. Sufian, J. Karpie, C. Egerer, K. Orginos, J.-W. Qiu, D. G. Richards, Pion Valence Quark Distribution from Matrix Element Calculated in Lattice QCD, Phys. Rev. D 99 (2019) 074507.

[84] M. B. Hecht, C. D. Roberts, S. M. Schmidt, Valencequark distributions in the pion, Phys. Rev. C 63 (2001) 025213.

[85] G. Grunberg, Renormalization Scheme Independent QCD and QED: The Method of Effective Charges, Phys. Rev. D 29 (1984) 2315.

[86] Y. L. Dokshitzer, Perturbative QCD theory (includes our knowledge of $\alpha(s)$ ) - hep-ph/9812252, in: Highenergy physics. Proceedings, 29th International Conference, ICHEP'98, Vancouver, Canada, July 23-29, 1998. Vol. 1, 2, 1998, pp. 305-324.

[87] B. Joó, J. Karpie, K. Orginos, A. V. Radyushkin, D. G. Richards, R. S. Sufian, S. Zafeiropoulos, Pion valence structure from Ioffe-time parton pseudodistribution functions, Phys. Rev. D 100 (2019) 114512.

[88] X. Gao, L. Jin, C. Kallidonis, N. Karthik, S. Mukherjee, P. Petreczky, C. Shugert, S. Syritsyn, Y. Zhao, Valence parton distribution of the pion from lattice QCD: Approaching the continuum limit, Phys. Rev. D 102 (9) (2020) 094513.

[89] P. Barry, JAM pion PDF analysis including resummation (Jun. 2020). doi:10.5281/zenodo.4019411.

[90] C. D. Roberts, Perspective on the origin of hadron masses, Few Body Syst. 58 (2017) 5. 